Southern Illinois University Edwardsville

SPARK

Pharmacy Faculty Research, Scholarship, and Creative Activity

2015

\title{
Survey Evaluation of Pharmacy Practice Involving Deaf Patients
}

McKenzie C. Ferguson

Southern Illinois University Edwardsville, mcfergu@siue.edu

Leah M. Shan

Follow this and additional works at: https://spark.siue.edu/pharmacy_fac

Part of the Health Communication Commons, and the Pharmacy and Pharmaceutical Sciences Commons

\section{Recommended Citation}

Ferguson, McKenzie C. and Shan, Leah M., "Survey Evaluation of Pharmacy Practice Involving Deaf Patients" (2015). Pharmacy Faculty Research, Scholarship, and Creative Activity. 1.

https://spark.siue.edu/pharmacy_fac/1

This Article is brought to you for free and open access by the School of Pharmacy at SPARK. It has been accepted for inclusion in Pharmacy Faculty Research, Scholarship, and Creative Activity by an authorized administrator of SPARK. For more information, please contact magrase@siue.edu,tdvorak@siue.edu. 
Title

Survey Evaluation of Pharmacy Practice Involving Deaf Patients 


\section{Abstract \\ Introduction}

For a patient that is deaf, providing patient care can be more difficult due to communication barriers. This study was conducted in order to better understand pharmacists' current means of communicating with deaf patients as well as investigating pharmacists' knowledge of their legal responsibility to these patients.

\section{Methods}

Surveys were used to gather information from pharmacists and were distributed in areas with a large population of deaf patients.

Results

Of the 73 pharmacists that completed surveys, 50 (68.5\%) of them interact with at least 1-5 deaf patients monthly. Pharmacists responded that accessibility of interpreters is the most significant barrier to communication and providing written material is the method most used to communicate with deaf patients. None of the 73 pharmacists who completed the survey felt that they have a legal obligation to provide and pay for an interpreter.

\section{Conclusion}

When interacting with a deaf patient, pharmacists may experience communication barriers. Pharmacists should strive to appropriately communicate with the deaf as well as familiarize themselves with legal obligations to this patient population.

Keywords: deaf, communication 


\section{INTRODUCTION}

Communication is a vital component of the interaction between a healthcare provider and a patient. If a provider cannot effectively relay important information to a patient, it can become much more difficult to achieve successful patient outcomes. This provider-patient communication can be significantly impeded if a patient is hearing impaired or deaf. ${ }^{1}$ There are more than 20 million people in the United States with hearing loss, and communication is one of the most significant obstacles to providing quality health care to these patients. ${ }^{2,3}$

Several studies show that language barriers lead to health disparities for deaf patients. ${ }^{4}$ The number of Americans with hearing loss is likely to increase as the population ages. As this patient population grows larger, it is imperative that health care providers learn to effectively communicate with patients who are deaf or hard-of-hearing. However, there is debate among healthcare providers regarding what effective communication entails. Some providers would constitute lip reading or writing notes as effective means of communicating health information; others believe that it is necessary for a sign language interpreter to be present in order to maintain effective provider-patient communication. ${ }^{1}$ However, one study showed that physicians only use sign language interpreters $20 \%$ of the time. ${ }^{5}$ When surveyed about communicating with their physicians, deaf patients responded that writing back and forth is the most common communication method they encounter. ${ }^{5}$ Surveys of physicians show that while they agree interpreters should be utilized, few actually have one present when interacting with a deaf patient. ${ }^{4}$ Studies also show that many physicians are not aware that they are legally obligated to provide interpreters. ${ }^{2,4}$ 
The American with Disabilities Act (ADA) mandates that health care providers offer an effective method of communication when interacting with deaf patients. Private healthcare providers (as well as state and local health care facilities) including pharmacies regardless of size are required to comply with ADA regulations. Auxiliary aids and services must be provided with a goal of providing equally effective communication as with people who do not have disabilities. ${ }^{6}$ For the deaf, auxiliary aids and services may include providing a qualified notetaker, a qualified sign language interpreter or other applicable interpreter, written materials, or access to technology such as videophones and hearing-aid compatible telephones among others. ${ }^{7}$ Video relay service is an option to Deaf patients to subscribe to so they can communicate through an interpreter via video who then verbalizes information to the other party. Video remote interpreting is another option but this requires accessing an off-site interpreter to provide real-time interpreting services. This may be useful in rural areas; however, it requires high-speed wireless and it relies on qualified interpreters being available at the time service is needed. ${ }^{7}$ In accordance with the ADA, if communication with the patient involves lengthy or difficult information, the provider has a legal obligation to provide an effective method of communication, which may include a qualified sign language interpreter, at no cost to the patient. ${ }^{8}$ Entities are required to give consideration to the requested service and specific needs of the patient but can also require "reasonable advance notice". Walk in requests for aids must be accommodated to the extent possible. ${ }^{7}$

Pharmacists face the same challenges as physicians and other healthcare providers when trying to communicate effectively with deaf patients about their medications. Without effective communication, pharmacists may not be able to provide proper medication 
counseling, and it is important for pharmacists to ensure deaf patients understand prescription directions and warnings about clinically significant adverse events. The purpose of this study was to investigate pharmacists' knowledge of how to interact with deaf patients and their current means of communicating with them. Another aim of the study was to determine if pharmacists working in areas with large populations of deaf patients are aware of their legal responsibility to these patients.

\section{METHODS}

This was a cross-sectional, survey-based study. The Southern Illinois University Edwardsville (SIUE) Institutional Review Board approved the study. Participation was voluntary and consent was provided by all participants.

Subjects

Subjects had to be at least 18 years of age or older and were also required to be a licensed pharmacist working in an area with a large deaf population. The investigators predetermined these areas after consulting Illinois Census statistics.

Study design

A 15 question survey was used to gather information from practicing pharmacists regarding their attitudes toward and methods of interacting with deaf patients. It was delivered either electronically or directly in-person to pharmacies located within the specified area as identified by census data. An Internet search was performed to locate pharmacies within the target geographical area.

The link to the online survey was sent to a list of preceptors affiliated with SIUE School of Pharmacy. One week after the original email was sent, a follow-up email was sent to the 
same list of preceptors. Paper versions of the survey were also delivered directly to pharmacies in areas with a large deaf population. The paper surveys were delivered to pharmacies in one visit, and pharmacists had the option of completing the survey immediately and returning it to the investigator or completing the survey at a later time and mailing the survey back with an addressed envelope provided by the investigator. Copies of the survey and addressed envelopes were provided to some pharmacies for off duty pharmacists to complete. Subjects were instructed not to put their names on the survey.

Descriptive statistics were used to evaluate the study data, including average, median, and mode. Graphs and tables were also created in order to compare data results.

\section{RESULTS}

A total of 73 pharmacists completed the survey. Because the exact total of how many surveys were distributed is unknown, a reliable response rate for the study surveys could not be determined. Twenty participants filled out the survey on SurveyMonkey ${ }^{\mathrm{TM}}$, and a total of 53 paper surveys were completed. Of these 73 pharmacists, 57 (78\%) responded that they currently work in a chain retail pharmacy, 14 (19\%) currently work in an independent retail pharmacy, and 2 (3\%) pharmacists responded 'other'. Table 1 shows approximately how many prescriptions the participants' respective pharmacies fill per day. A majority (93\%) of the participating pharmacists responded that they had previously interacted with a deaf patient; only 5 (7\%) of the 73 pharmacists responded that they had never had an encounter with a patient who was deaf. Table 2 shows how many interactions the pharmacists have with deaf patients on a monthly basis. 
When asked how difficult it was to communicate with a deaf patient, 52 (74\%) pharmacists responded that it was slightly difficult. Eleven (16\%) of the pharmacists responded not difficult, and $7(10 \%)$ of pharmacists responded that it was very difficult to communicate with a deaf patient. Pharmacists were also asked to rank their comfort level in working with deaf patients on a scale of 1-10 with 1-2 being very uncomfortable and 9-10 being very comfortable (Table 3). Even though they work in areas with a large deaf population, only 22 (30\%) pharmacists responded that they were somewhat comfortable or very comfortable when working with deaf patients. Seven (9.5\%) of the pharmacists responded that they are very uncomfortable when working with deaf patients.

When asked if they felt pharmacists have a legal obligation to ensure effective communication to deaf patients, 65 (89\%) of the study participants responded "yes", while 8 (11\%) responded "no". Study participants were provided a list of responses and were asked to identify from the list what a pharmacist's legal obligation may include. Participants were instructed to only select one option while taking the survey (Figure 1). A majority of the study participants felt that their legal obligation to deaf patients includes providing written materials (79.5\%) to the patient. In a separate question that asked what a pharmacist' legal obligation may include nine (12\%) pharmacists responded they would provide an interpreter upon patient request, and $6(8 \%)$ pharmacists responded there is no legal obligation for pharmacists to ensure effective communication. Of the 73 pharmacists who completed the survey, none felt that a pharmacist's legal obligation to deaf patients includes providing and paying for an interpreter or providing an interpreter if the patient's insurance will cover the cost. 
The pharmacists were also asked which methods they have used or would use in order to communicate with a deaf patient (Figure 2). Participants were allowed to select all applicable methods. Writing information out for the patient was the most selected method; only 1 pharmacist out of 73 did not select it as a method he or she would use to communicate with a deaf patient. Speaking so the patient can read your lips (64\%) and use of a family member to interpret information (55\%) were the next most commonly used methods of communication.

The study participants were provided with a list of barriers to communication with patients who are deaf and were asked to rank these seven barriers from 1 (most significant) to 7 (least significant). Each rank given to a certain barrier was then tallied, and the average rank for each communication barrier was then calculated (Table 4). Accessibility of an interpreter was shown to be the most significant barrier with the lowest average rank, and limited understanding of Deaf Culture was shown to be the least significant barrier.

When questioned about providing care to deaf patients, 26 (36\%) of the pharmacists answered they believe that deaf patient receive 'less than their best' care due to communication barriers that exist. The other 47 (64\%) participants reported that they do not believe deaf patients receive 'less than their best' care.

When asked if they had employer support to utilize resources for improving communication with deaf patients, the response from the study participants was almost even. Thirty-four (47\%) of the pharmacists responded that they do have employer support, while 39 (53\%) responded that they do not have employer support to utilize resources. The pharmacists who felt they have employer support were then questioned about the resources that they can 
access at work in order to communicate with deaf patients. Though the survey instructions told pharmacists who answered yes to having employer support (34) to answer question \#15, 39 pharmacists answered survey question \#15, and 36 (92\%) of them responded that their employers provide access to written materials in order to communicate with deaf patients. Only 1 (2.6\%) pharmacist responded that he or she had access to an interpreter (Figure 3).

\section{DISCUSSION}

Studies have shown that communication barriers exist when deaf and hard-of-hearing patients are receiving healthcare, and these barriers can result in misunderstandings and incomplete care. ${ }^{1,2,4}$ These studies also highlight that not all health care providers have a good understanding of Deaf Culture, and they are not always completely aware of their legal obligations to deaf patients. ${ }^{1-3}$ However, most of the current literature discusses physicians' interactions with deaf patients; there is limited literature available regarding pharmacists' interactions with deaf patients. This lack of understanding and impact on communication greatly impacts the quality of care that deaf patients receive and may lead to distrust of the pharmacist, medication misuse, and less than optimal health outcomes as a result.

The results of this study show that there is room for improvement during interactions between pharmacists and deaf patients. Even though most of the participating pharmacists have at least a monthly encounter with a deaf patient, only $30.5 \%$ stated that they are comfortable interacting with deaf patients. Pharmacists also do not seem to be aware of their legal obligations to deaf patients. According to the ADA, the healthcare provider is responsible for paying the cost of hiring an interpreter. ${ }^{2,6,8}$ However, of the 73 pharmacists who completed the survey, not a single one responded that he or she has a legal obligation to provide and pay 
for an interpreter. Though we did not ask pharmacists about their legal obligation to counsel all patients, Illinois state law does mandate that pharmacists offer to counsel on all prescriptions. In addition, Illinois state law states that if oral counseling is not practicable for the patient, alternative forms of information should be provided, but it is in relation to later oral consultation. $^{9}$

There are several methods for communicating with deaf patients including American Sign Language, use of a qualified interpreter, reading lips, writing information, use of a telecommunications device, use of a family member to interpret, and using the Internet. Though it varies by state, in the state of Illinois, interpreters are required to be licensed based on proficiency level and only advanced or master level interpreters can communicate all medical information. ${ }^{9}$ Interpreters are liable for complying with regulations related to protected health information and the Health Insurance Portability and Accountability Act (HIPAA) providing accurate translation of information without imposing judgment. Nearly $99 \%$ of the pharmacists in this study responded that providing written information for the patient is a method they have used, while only $22 \%$ have used or would use an interpreter in order to communicate with deaf patients. Providing written materials may be sufficient with some patients; however, studies have shown that even though deaf patients have normal cognitive abilities, it is possible for them to have impaired reading and writing skills. ${ }^{5}$ Therefore, deaf patients may not always understand what providers are trying to communicate in writing, which can lead to misunderstandings of treatment and inadequate medication counseling. ${ }^{3}$ Moreover, the patient may be too intimidated to ask the provider to explain a word that he or she does not understand. 
This study shows that pharmacists are not fully aware of their legal obligations to deaf patients. Most pharmacists felt that writing down information for the patient was a sufficient method of communication. However, pertinent information may be left out because writing it all out can be time consuming, and this does not ensure the patient understands. Future studies need to be done in order to evaluate different methods of educating pharmacists on their legal obligations to deaf patients including knowledge of devices and methods such as video relay services. Studies should also be conducted to determine what deaf patients feel are the biggest barriers to communication between them and their pharmacists.

The findings of this study have several limitations. Since the study participants were limited to small geographical area, the responses may not be representative of all pharmacists. It is possible that pharmacists in different areas may interact differently with deaf patients. Even though the study participants work in areas with a large deaf population, the number of encounters with deaf patients varied between the pharmacists who took the survey. Also, it is possible that pharmacists' responses were affected by the way the surveys were distributed. The pharmacists who completed the survey online could take the survey at any time and not feel rushed; conversely, the pharmacists who completed the paper surveys were at work when asked to take the survey.

\section{CONCLUSION}

Communication is a vital part of providing quality healthcare. As a healthcare provider, pharmacists have a responsibility to ensure that medication related information is effectively conveyed to deaf patients. This study found that many pharmacists prefer to communicate with deaf patients by providing written materials, but depending on the patient, this might not 
always be an effective way to communicate important medication information. It was also found that pharmacists are not aware of their legal obligation to provide and pay for an interpreter. In order to provide the best care possible, both pharmacists and their employers should strive to eliminate communication barriers between themselves and deaf patients. 


\section{REFERENCES}

1. Iezzoni LI, O’Day BL, Killeen M, et al. Communicating about health care: observations from persons who are deaf or hard of hearing. Ann Intern Med. 2004 Mar 2;140(5):35662.

2. Scheier, DB. Barriers to health care for people with hearing loss: A review of the literature. J NY State Nurses Assoc. 2009 Spring-Summer;40(1):4-10.

3. Ralston E, Zazove P, Gorenflow DW. Physicians' attitudes and beliefs about deaf patients. J Am Board Fam Pract. 1996 May-Jun;9(3):167-73.

4. Hoang L, LaHousse SF, Nakaji MC, et al. Assessing Deaf cultural competency of physicians and medical students. J Canc Educ. 2011;26:175-182.

5. Ebert DA, Heckerling PS. Communication with deaf patients. Knowledge, beliefs, and practices of physicians. JAMA. 1995 Jan 18;273(3):227-9.

6. ADA.gov [Internet]. ADA: Title III Highlights. [cited 2014 August 21]. Available from: http://www.ada.gov/t3hilght.htm

7. ADA.gov [Internet]. ADA Requirements: Effective Communication. [updated 2014 Jan 31; cited 2014 August 21]. Available from: http://www.ada.gov/effective-comm.htm

8. ADA.gov [Internet]. ADA Business Brief: Communicating with people who are Deaf or hard of hearing in hospital settings. [updated 2012 Jan 12; cited 2012 Jan 13]. Available from: http://www.ada.gov/hospcombrprt.pdf 
9. Joint Committee on Administrative Rules, Administrative Code. Part 1330 Pharmacy

Practice Act; Section 1300.700 Patient Counseling. [cited 2014 August 21]. Available from: http://www.ilga.gov/commission/jcar/admincode/068/068013300G07000R.html

10. Illinois Deaf and Hard of Hearing Commission. [cited 2014 August 21]. Available from:

http://www2.illinois.gov/idhhc/Documents/IDHHC\%20Publications/Level\%20Brochure2

.pdf 


\section{TABLES}

Table 1: Approximate daily prescriptions filled by pharmacy.

\begin{tabular}{|l|c|}
\hline Number of prescriptions filled daily & $\begin{array}{c}\text { Response (\%) } \\
\mathbf{N}=73\end{array}$ \\
\hline$<250$ & $23(31 \%)$ \\
\hline $250-500$ & $32(44 \%)$ \\
\hline $500-750$ & $13(18 \%)$ \\
\hline$>750$ & $5(7 \%)$ \\
\hline
\end{tabular}


Table 2: Number of interactions with deaf patients per month.

\begin{tabular}{|l|c|}
\hline Interactions per month & $\begin{array}{c}\text { Response (\%) } \\
\mathbf{N = 7 3}\end{array}$ \\
\hline Never & $13(18 \%)$ \\
\hline $1-5$ & $50(68.5 \%)$ \\
\hline $5-10$ & $4(5.5 \%)$ \\
\hline$>10$ & $6(8 \%)$ \\
\hline
\end{tabular}


Table 3: Comfort level in working with deaf patients.

\begin{tabular}{|l|c|}
\hline Comfort level rank & $\begin{array}{c}\text { Response (\%) } \\
\mathbf{N}=73\end{array}$ \\
\hline 1-2 Very uncomfortable & $7(9.5 \%)$ \\
\hline 3-4 Slightly uncomfortable & $16(22 \%)$ \\
\hline 5-6 Neutral & $28(38 \%)$ \\
\hline 7-8 Somewhat comfortable & $7(9.5 \%)$ \\
\hline 9-10 Very comfortable & $15(21 \%)$ \\
\hline
\end{tabular}


Table 4: Barriers to communication with patients who are deaf.

\begin{tabular}{|l|c|}
\hline Communication barriers* & $\begin{array}{c}\text { Response } \\
\text { Average }\end{array}$ \\
\hline Accessibility of an interpreter & 2.97 \\
\hline Patient relies on American Sign Language for communication & 3.11 \\
\hline Patient acting like he/she understands, but does not understand & 3.36 \\
\hline Reading level of deaf patient & 3.97 \\
\hline Incomplete communication when providing information by writing & 4.42 \\
\hline Lack of employer resources to effectively communicate & 5.12 \\
\hline $\begin{array}{l}\text { Limited understanding of Deaf culture (i.e. offending or frustrating the } \\
\text { patient) }\end{array}$ & 5.17 \\
\hline
\end{tabular}

*Study participants were asked to rank each barrier from 1 to 7 , with 1 being the most significant barrier and 7 being the least significant barrier. 


\section{FIGURES}

Figure 1: Pharmacists' perception of their legal obligation to deaf patients.

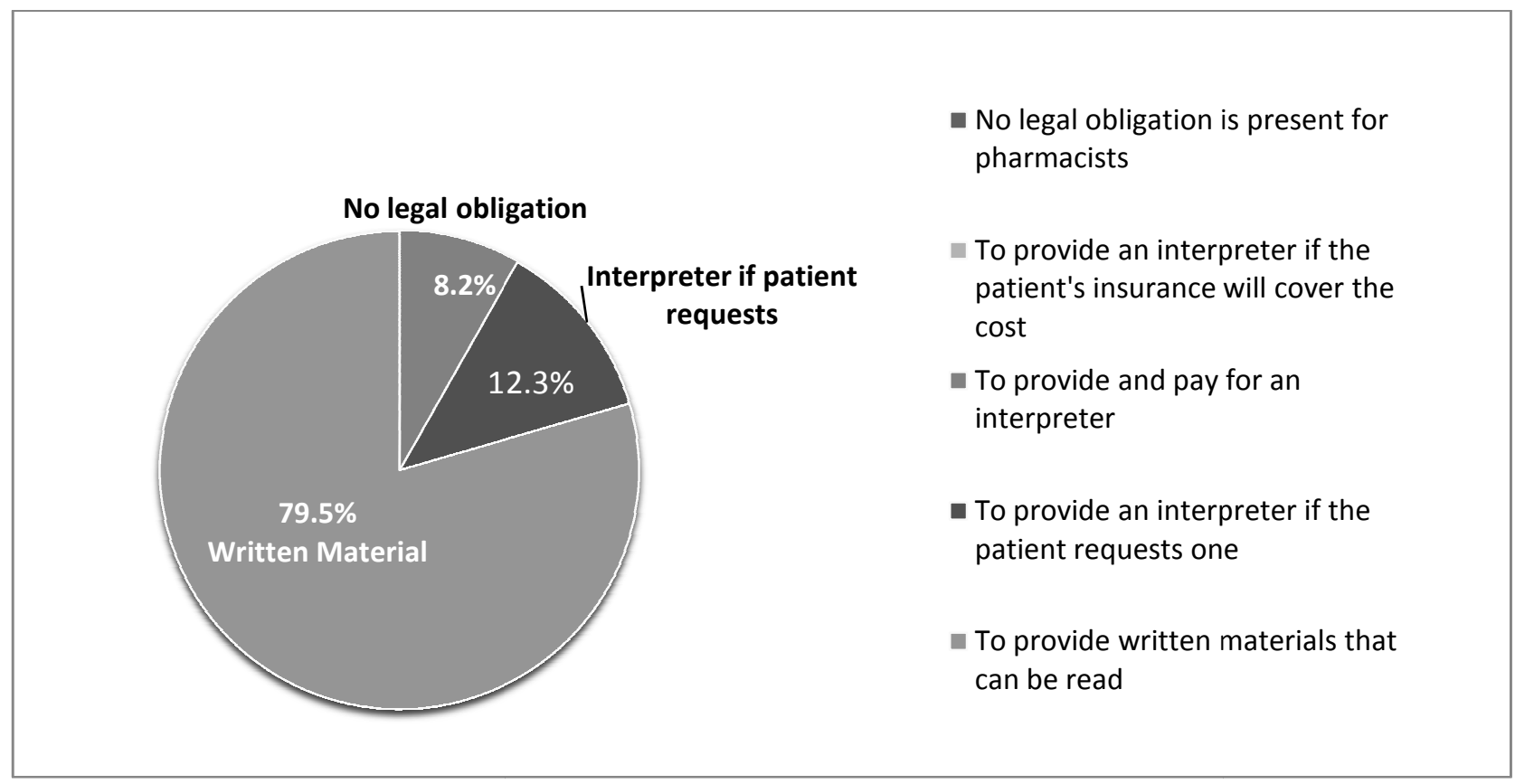


Figure 2: Methods pharmacists have used (or would use) in order to communicate with a deaf

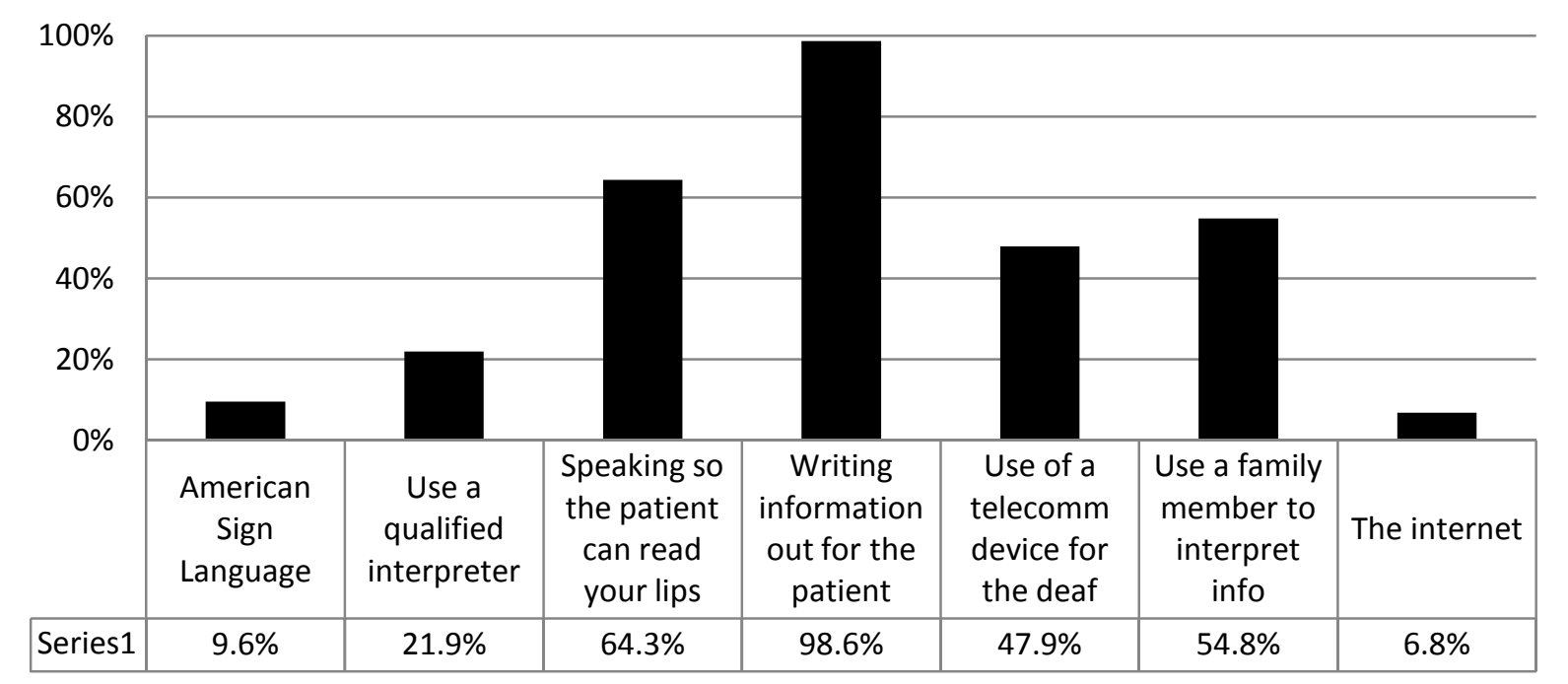


Figure 3: Resources accessible to pharmacists for communication with deaf patients.

(Select all that apply)

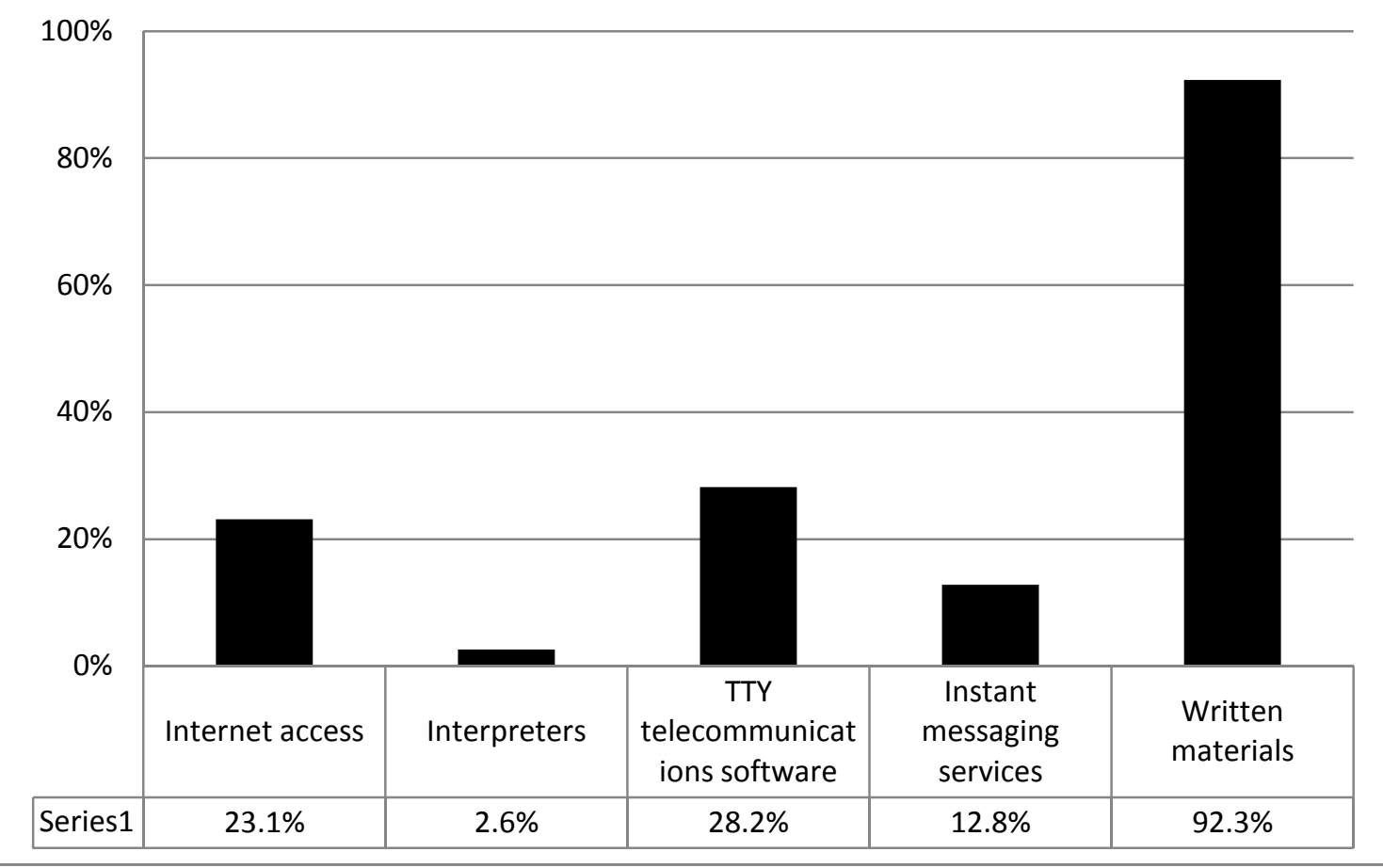

